\title{
The science of symmetry breaking: A personal journey
}

\section{Harold T Stokes}

Brigham Young University, Provo, United States of America

stokesh@byu.edu

Over the past 40 years, the use of group representation theory has transformed the study of phase transitions in crystalline materials. From my own perspective, I will present the history and development of the innovative methods and computational infrastructure that have supported this transformation. Highlights will include (1) the tabulation of irreducible representations for crystallographic space groups and their superspace extensions, (2) the determination of isotropy subgroups, (3) the projection and parameterization of symmetry modes, (4) the tabulation of superspace symmetry groups, (5) and the development of the online ISOTROPY Software Suite, which makes all of these advances and data sources immediately and freely accessible to the international research community.

Keywords: representation theory, phase transitions 